\title{
Microglial depletion under thalamic hemorrhage ameliorates mechanical allodynia and suppresses aberrant axonal sprouting
}

\author{
Shin-ichiro Hiraga, ${ }^{1}$ Takahide Itokazu, ${ }^{1,2,3}$ Maki Hoshiko, ${ }^{1,2}$ Hironobu Takaya, ${ }^{1}$ Mariko Nishibe, ${ }^{1,4}$ \\ and Toshihide Yamashita $a^{1,2,3,5}$ \\ 'Department of Molecular Neuroscience, Graduate School of Medicine, Osaka University, Suita, Osaka, Japan. ${ }^{2}$ WPI \\ Immunology Frontier Research Center, Osaka, Japan. ${ }^{3}$ Department of Neuro-Medical Science, Graduate School of Medicine, \\ ${ }^{4}$ Office of Strategic Innovative Dentistry, Graduate School of Dentistry, Osaka University, Suita, Osaka, Japan. ${ }^{5} \mathrm{Graduate}$ \\ School of Frontier Biosciences, Osaka University, Osaka, Japan.
}

Central poststroke pain (CPSP) is one of the neuropathic pain syndromes that can occur following stroke involving the somatosensory system. However, the underlying mechanism of CPSP remains largely unknown. Here, we established a CPSP mouse model by inducing a focal hemorrhage in the thalamic ventrobasal complex and confirmed the development of mechanical allodynia. In this model, microglial activation was observed in the somatosensory cortex, as well as in the injured thalamus. By using a CSF1 receptor inhibitor, we showed that microglial depletion effectively prevented allodynia development in our CPSP model. In the critical phase of allodynia development, c-fos-positive neurons increased in the somatosensory cortex, accompanied by ectopic axonal sprouting of the thalamocortical projection. Furthermore, microglial ablation attenuated both neuronal hyperactivity in the somatosensory cortex and circuit reorganization. These findings suggest that microglia play a crucial role in the development of CPSP pathophysiology by promoting sensory circuit reorganization.

Conflict of interest: The authors have declared that no conflict of interest exists.

Copyright: () 2020, American Society for Clinical Investigation.

Submitted: July 15, 2019

Accepted: December 30, 2019

Published: February 13, 2020

Reference information: /CI Insight. 2020;5(3):e131801.

https://doi.org/10.1172/jici.

insight.131801.

\section{Introduction}

Central poststroke pain (CPSP), occurring following ischemic and hemorrhagic stroke, is categorized as a neuropathic pain syndrome. CPSP occurs after lesions at any level of the sensory pathway in the CNS, including the thalamus, cortex, and medulla, and the prevalence of CPSP in stroke patients is $1 \%-12 \%$ (1). In particular, patients who suffer a hemorrhagic injury encompassing the ventroposterior part of the thalamus have a higher chance of developing CPSP $(1,2)$. CPSP is characterized by allodynia, hyperalgesia, and spontaneous dysesthesia. In many cases, these symptoms persist for years and are extremely difficult to treat $(1,3)$.

Although the underlying mechanism of CPSP remains largely unknown, maladaptive network reorganization is considered a key feature $(4,5)$. Based on clinical and experimental observations, various abnormal network models have been proposed to explain the development of CPSP, and many of them emphasize the importance of hyperactivity of the thalamocortical circuit $(1,5)$. However, neuroanatomical evidence of neural circuit reorganization is lacking.

In the last decade, microglia have emerged as central players in the development of neuropathic pain (6). Microglia are macrophage-like cells in the CNS and are primarily responsible for the inflammatory response (7). However, recent evidence indicates that microglia have a variety of physiological, noninflammatory functions that are crucial for brain development, CNS maintenance, and neuroplasticity (8-14). Moreover, microglia have been implicated as contributors to the disease process in many CNS disorders, such as neurodegenerative disease, psychiatric disorder, and neuropathic pain $(11,15,16)$. In the diseased state, microglia have complex roles in disease pathogenesis, including the inflammatory response, debris clearance, synaptic pruning, and neuronal excitation (11, 16-18). Importantly, minocycline, one of the modulators of microglial reactivity, has been shown to ameliorate CPSP symptoms transiently (19). However, microglial involvement in the pathophysiology of CPSP remains largely unknown. 
In the present study, we examined the possible involvement of microglia in the development of CPSP. By employing a focal thalamic hemorrhage (TH) model in mice, we identified local microglial activation within the sensorimotor cortex, as well as in the injured thalamus. Then, we assessed the effect of pharmacological ablation of microglia on TH and found that the treatment completely prevented the development of TH-induced allodynia. Moreover, to elucidate the mechanism of allodynia development, we examined the thalamocortical projection in our TH model and revealed the occurrence of aberrant axonal rewiring in the somatosensory cortex. In addition, we demonstrated that anatomical reorganization was attenuated by microglial depletion.

\section{Results}

Establishment of the CPSP mouse model. To elucidate the mechanism of CPSP, we developed a focal TH model in mice by injecting collagenase into the ventral posterolateral nucleus (VPL) of the thalamus, the relay nucleus of the somatosensory pathway (Figure 1A). We carefully tested the injection volume, speed, and coordinates and established a highly reproducible injection method to confine the lesion area to the ventrobasal complex (VB), which is composed of the VPL and ventral posteromedial nucleus (VPM) (see Methods and Figure 1, A-C).

Next, to examined whether our TH model developed pain-like behavior, we tested mechanical allodynia by measuring the hind paw withdrawal response to von Frey filament stimulation (Figure 1D). To evaluate aversive behaviors, a response is considered positive when the mouse shows nocifensive behaviors, including licking or flicking of the paw (20). As expected, the withdrawal threshold of the contralesional hind paw was significantly decreased 5 days after $\mathrm{TH}$ induction and persisted throughout the testing period (Figure 1E) compared with the ipsilesional paw (Supplemental Figure 1A; supplemental material available online with this article; https://doi.org/10.1172/jci.insight.131801DS1). We also checked the motor performance of TH mice by the 4-point-scale neurological scoring method, ladder walk test, and rotarod test (Figure 1, F-H); no apparent motor deficit was observed. On neurological scoring, the neurological grade was not different between groups (Figure 1F). On the ladder walk test, the number of slips of contralesional paws (Figure 1G) or ipsilesional paws (Supplemental Figure 1B) showed no significant increase. Additionally, on the rotarod test, there was no difference in the time to fall from the rotating rod (Figure $1 \mathrm{H}$ ).

To assess whether the development of mechanical allodynia was accompanied by the exacerbation of injury, we quantified the lesion volume in Nissl-stained serial sections on days 4, 7, and 21 after TH (Figure 1 , I and J). No enlargement of injury was observed over time, suggesting that allodynia development was not related to the expansion of the damaged area.

TH mice exhibit microglial activation in the ipsilesional primary somatosensory cortex, as well as in the injured thalamus. Microglia are reportedly involved in the development of neuropathic pain in several rodent models (21-24). Thus, we examined whether microglial cells were activated in our TH model along the sensory pathway (thalamus, spinal cord, and primary somatosensory cortex [S1]). Consistent with previous reports $(19,24)$, microglial accumulation was observed in the lesioned thalamus on posthemorrhage days 1, 4, and 7. Morphologically, these microglia showed hypertrophy of the cell body and shortening of the protrusion, which are typical morphological features of activated microglia (Figure 2A). Interestingly, activated microglia were also observed in the ipsilesional S1 on posthemorrhage days 1 and 4 (Figure 2B); thereafter, at day 7, very few microglia were activated. The contralesional $\mathrm{S} 1$ showed no signs of microglial activation throughout the observation period (Figure $2 \mathrm{~B}$ ), and in the spinal cord, ionized calcium binding adaptor molecule 1 (Iba-1) reactivity remained unchanged on both sides (Figure 2C). Moreover, mRNA quantification of microglia-related genes also suggests microglial activation in the affected thalamus and S1 (Supplemental Figure 2, A and B) but not in the spinal cord (Supplemental Figure 2C). These findings motivated us to focus on the role of limited time activation of microglia in the ipsilesional S1.

Microglial depletion attenuates the development of mechanical allodynia induced by TH. To investigate the role of microglia in the development of pain-like behavior, we depleted microglia in TH mice by orally administrating PLX3397, a selective CSF1 receptor (CSF1R) inhibitor (25). Mice were treated with chow containing PLX3397 in the following time periods to elucidate the crucial period of microglial involvement in the development of TH-induced pain: (a) starting at 21 days before $\mathrm{TH}$ induction until 21 days after TH (Group 1, $n=5$ ), (b) from 7 days to 21 days after TH (Group 2, $n=4$ ), (c) from day 0 to 21 days after TH (Group 3,n=4), and (d) from 21 days before to 7 days after TH (Group 4,n=3). 


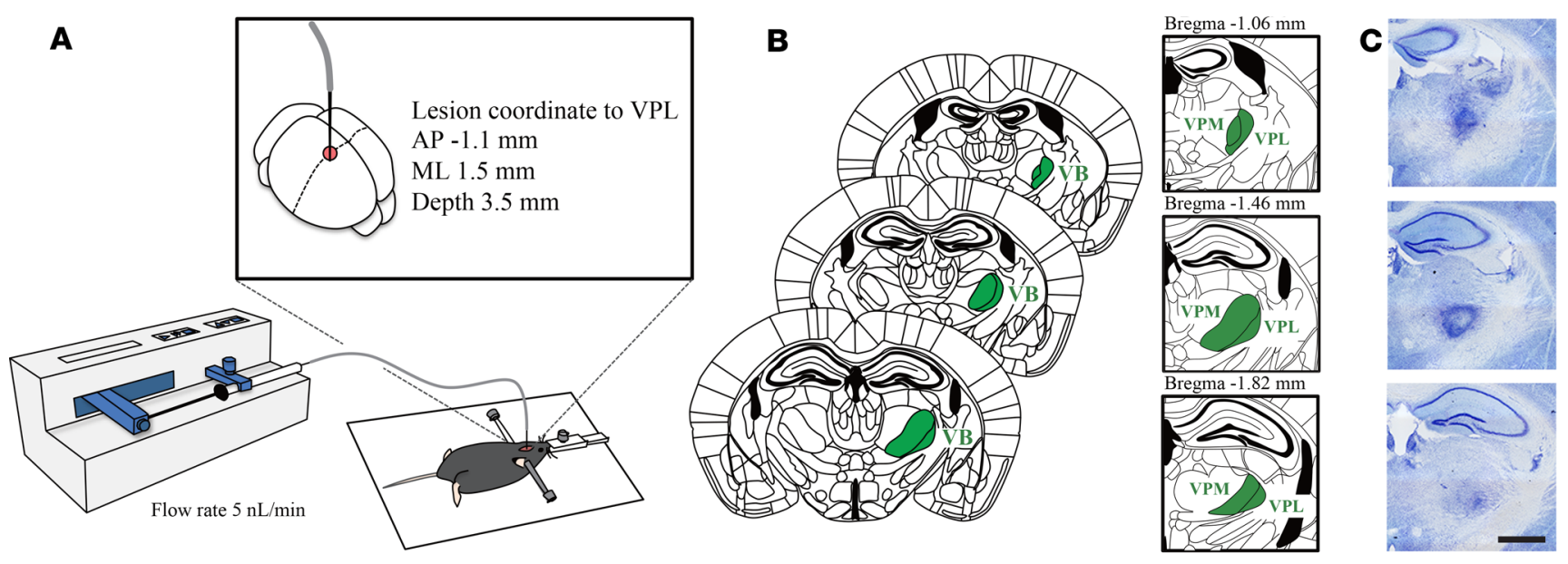

D $\quad \nabla$ Thalamic hemorrhage induction $\mid$ von Frey test $\nabla$ Neurologic examination, Ladder walk test, Rotarod test
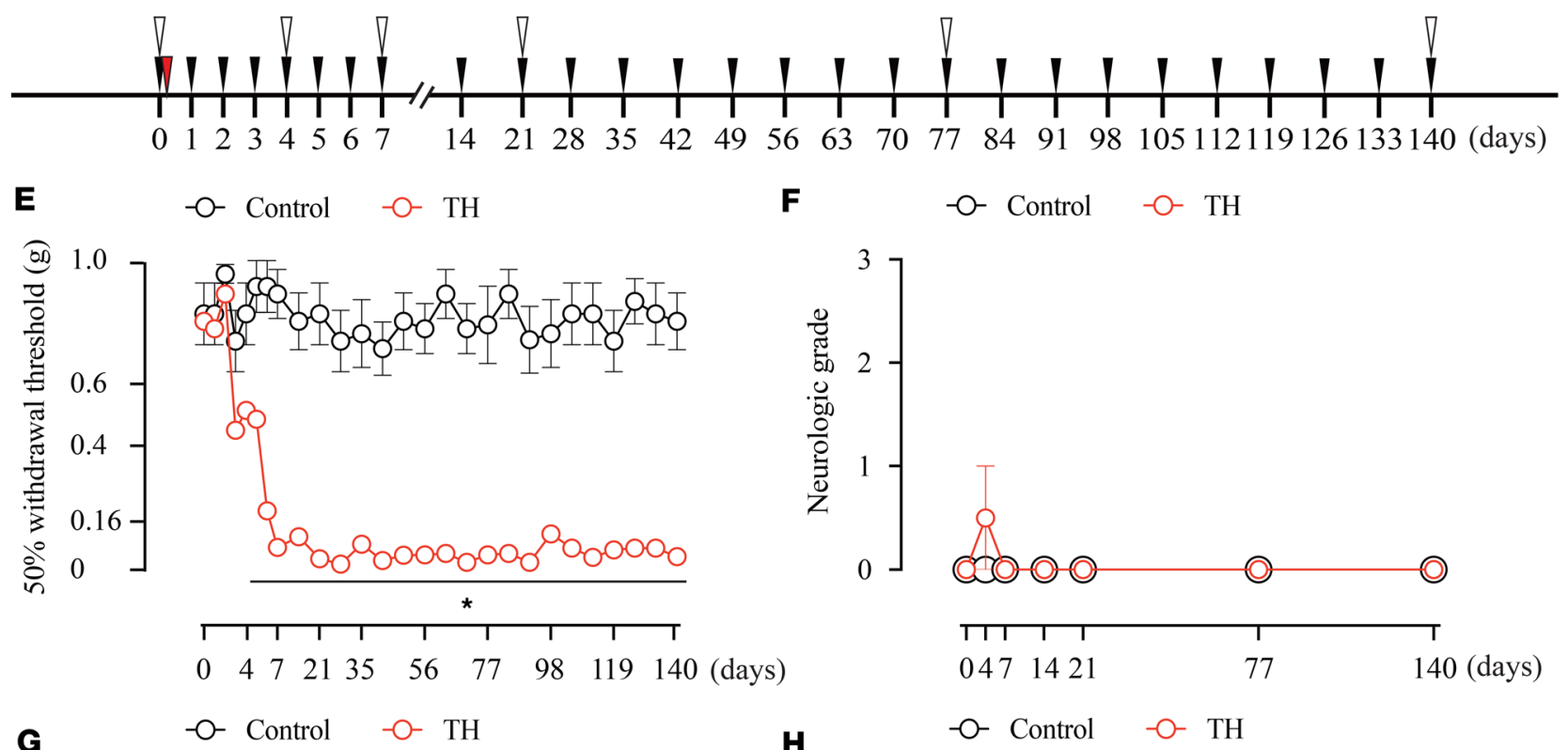

F $\quad-$ - Control $\quad-\mathrm{O}$ TH
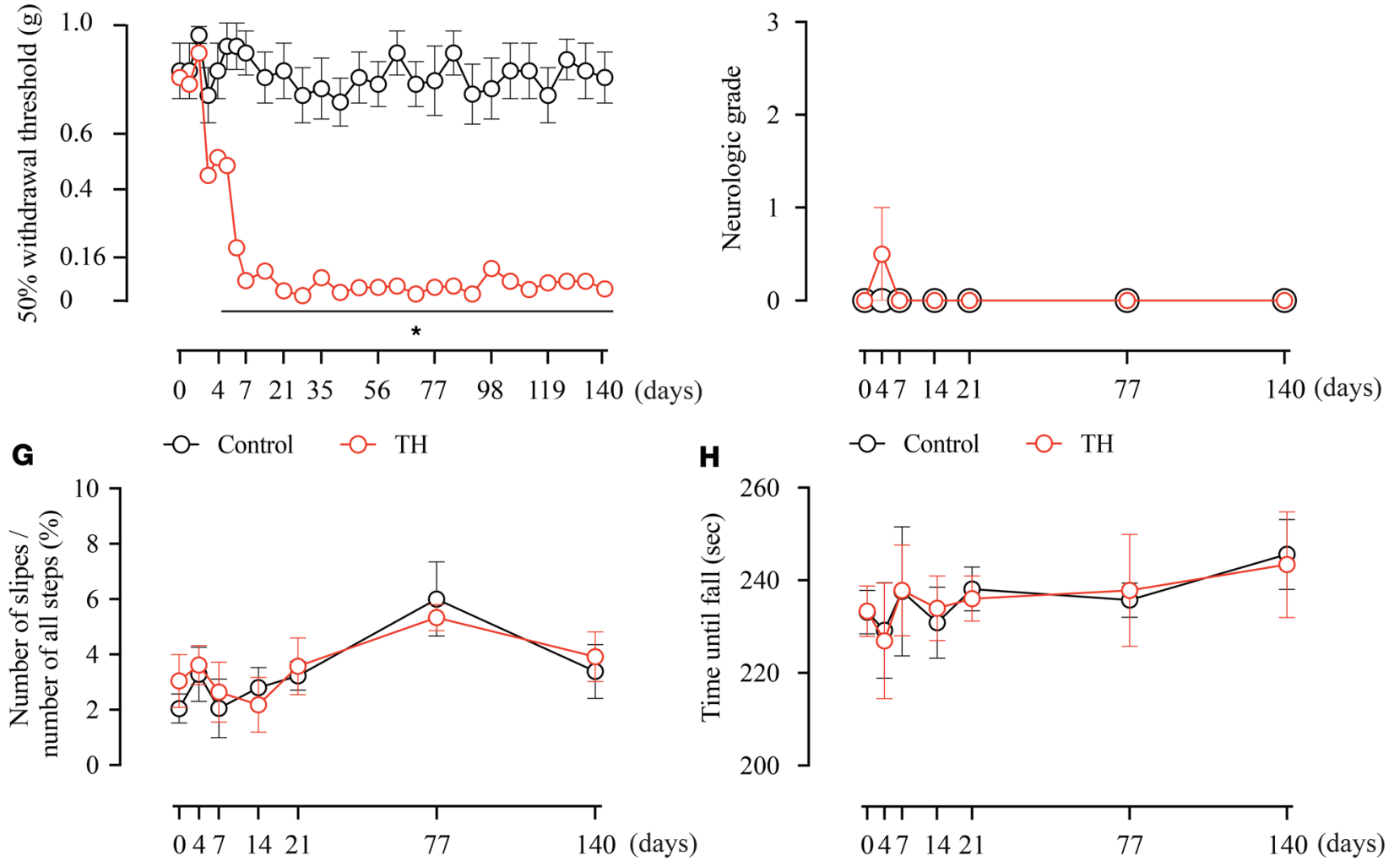

I Day 4

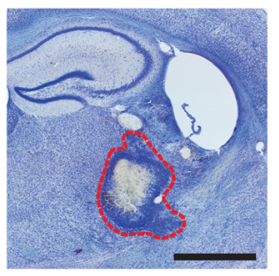

Day 7

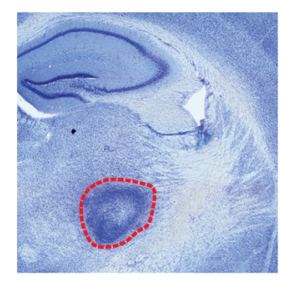

Day 21

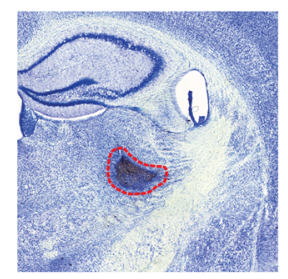

$\mathbf{J}$

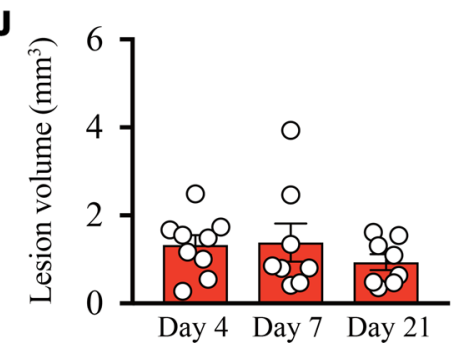


Figure 1. Thalamic hemorrhage induces mechanical allodynia without affecting motor functions. (A) Schema of the thalamic hemorrhage (TH) induction method. VPL, ventral posterolateral nucleus; AP, anterior-posterior; ML, medial-lateral. (B) Anatomy of our injection target, the VPL, and its surrounding area. The ventrobasal complex (VB) consists of the VPL and ventral posteromedial nucleus (VPM). (C) Representative image of the Nissl-stained tissue sections. Scale bar: $1 \mathrm{~mm}$. (D) An experimental design showing the time-course of TH induction and behavioral tests. (E) The $50 \%$ withdrawal threshold of the contralesional hind paw was significantly decreased 5 days after TH induction and persisted throughout the testing period $\left({ }^{*} P<0.01,2\right.$-way repeated measures ANOVA followed by Sidak's multiple comparisons test). (F-H) No motor deficit was observed in TH mice. (F) The neurologic grade $(P>0.05$, group effect by 2-way repeated measures ANOVA). (C) The ladder walk test $(P>0.05$, group effect by 2 -way repeated measures ANOVA). (H) The rotarod assessment $(P>0.05$, group effect by 2 -way repeated measures ANOVA). (I and J) There was no significant difference in the lesion area quantified on days 4,7 , and 21 after TH ( $P>0.05$, group effect by 1-way repeated measures ANOVA). Red outlines in images represent the edges of lesions. Scale bar: $1 \mathrm{~mm}$.

First, we confirmed the efficiency of microglial depletion by IHC and flow cytometry (Figure 3A). As expected, PLX3397 treatment resulted in a drastic decrease in Iba-1 reactivity (Figure 3B). Flow cytometry experiments also showed a significant reduction in the number of (CX3CR $\left.1^{\text {hi }}\right)$ microglia on postlesion days 7 and 21 in the 4 PLX-treated groups (Figure 3, C and D) compared with that in the no-PLX treatment TH groups. Based on the previous reports, the effects of PLX treatment on peripheral circulating CSF1Rpositive myeloid cells are known to be limited (25-28). We also confirmed the number of infiltrating macrophages and neutrophils after PLX treatment (Supplemental Figure 4, A and B). Consistent with previous reports, although over $99.5 \%$ of microglia were eliminated, PLX treatment had only minor effects on the number of infiltrating macrophages and neutrophils.

Next, we examined the effect of microglial depletion on allodynia development by the von Frey filament test (Figure 3E). The withdrawal threshold in PLX-treated Group 1 returned to that observed in the no-hemorrhage Control group, suggesting the resolution of hyperalgesia by PLX treatment. Alternatively, PLX treatment from day 7 posthemorrhage (Group 2) was unable to reverse the withdrawal threshold decrease. Similarly, starting PLX administration just after TH induction (Group 3) was insufficient to attenuate the development of allodynia. In contrast, when PLX was applied starting at 21 days before TH induction, mechanical allodynia following TH was completely abolished, and the effect lasted for at least 2 weeks after we stopped PLX administration (Group 4). Considering the possibility that sex difference may affect the behavioral outcome, we tested the effect of TH with and without PLX treatment on female mice (Supplemental Figure 3A). In our CPSP model, we found no evidence of sex differences; TH-induced female mice also developed allodynia, and PLX treatment effectively attenuated this (Supplemental Figure 3B). These results suggest that microglial cells are critically involved in allodynia development, especially in the acute phase after hemorrhage.

Microglial depletion does not affect the lesion volume. To examine the possibility that the PLX3397 treatment reduced the hemorrhage lesion to ameliorate the neurological symptoms, we measured the lesion volume at several time points (Supplemental Figure 5). However, the lesion volume was not significantly different among any of the groups (Supplemental Figure 5, A-C). Therefore, PLX3397 did not affect the lesion volume in our experimental paradigm.

Branch formation from thalamocortical neurons into layer 4 of the S1 after TH. In a recent study, genetic ablation of the VB in the developmental stage resulted in anatomical and functional rewiring of the afferent projection from the posterior nucleus (Po), which conveys tactile information to layers 1 and $5 \mathrm{a}$ ( $\mathrm{L} 1$ and L5a) neurons of the S1, onto L4 neurons in the S1 (29). However, the possible role of such axonal rewiring in the CPSP has not been studied thus far. To address this issue, we directly visualized Po axons in Control mice and TH mice by injecting biotinylated dextran amines (BDA) into the Po (Figure 4, A-C). As shown in Figure 4D, Control Po axons were mainly distributed in L5a and rarely observed in L4 in the S1. However, in TH mice, Po axons branched into L4, suggesting the occurrence of aberrant axonal sprouting induced by TH (Figure 4D, middle panel). Based on the observation that microglia were activated in the ipsilesional S1 (Figure 2B), we next examined whether PLX treatment could suppress the formation of aberrant rewiring. As expected, this ectopic projection was no longer observed in PLX-treated mice (Figure 4D, lower panel). To confirm this result, we quantified the BDA-labeled axon terminal by its florescent intensity (pixel per $\mathrm{mm}^{2}$ ) in each layer (L1-L6) of the S1 (Figure 4E). Axon density in L4 was significantly increased by TH induction, whereas the increase was completely abolished in the PLX-treated group. These results indicate that $\mathrm{TH}$ triggers the occurrence of an unusual axonal projection from Po to L4 of the $\mathrm{S} 1$ and that this aberrant projection is reversed by microglial depletion.

Microglial depletion prevents ectopic neuronal activity in the S1 after TH. After demonstrating that microglial depletion abolishes the aberrant rewiring in the $\mathrm{S} 1$, we next examined whether this anatomical alteration is paralleled by neuronal activity. For this purpose, we analyzed c-fos expression in the S1 by IHC on day 

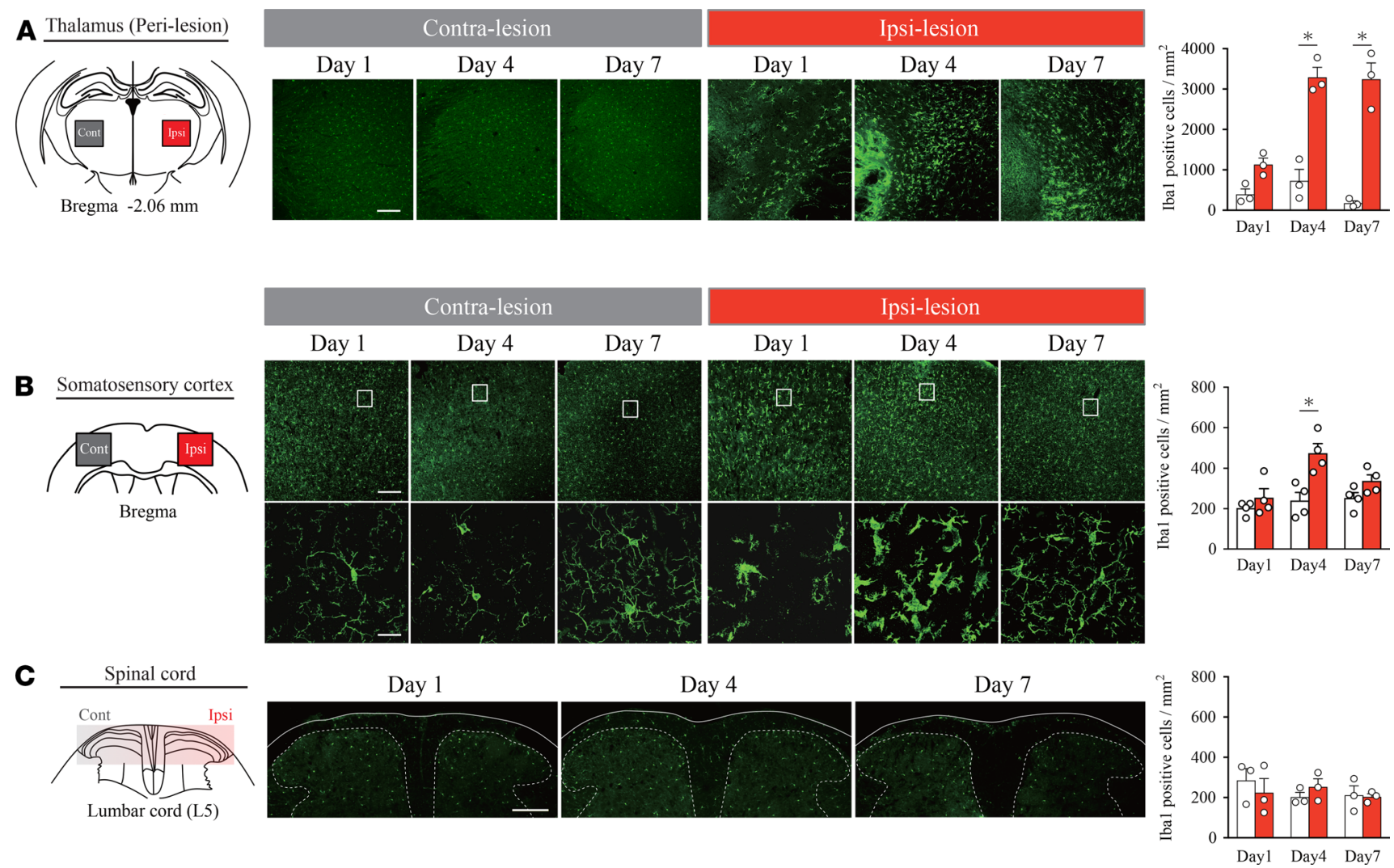

Figure 2. Microglia are activated in the perithalamic lesion sites and ipsilesional S1. (A) Iba-1 immunostaining revealed microglial activation in the perilesional area on days 1, 4, and 7. Iba-1-positive cells in the perilesional area in the TH group were increased on days 4 and 7 compared with those in the Control ${ }^{*} P<0.01$, 2-way ANOVA followed by Sidak's multiple comparisons test). Scale bar: $200 \mu \mathrm{m}$. (B) Morphological changes in microglia were also observed in the ipsilesional S1 on posthemorrhage days 1 and 4. Microglial activation was diminished on day 7 compared with that on day 4. Contralesional S1 showed no signs of microglial activation on posthemorrhage days 1,4 , and 7 . Iba-1-positive cells in the ipsilesional 51 were increased on day 4 compared with those in the Control $\left({ }^{*} P<0.01\right.$, 2-way ANOVA followed by Sidak's multiple comparisons test). Scale bars: $200 \mu \mathrm{m}$ (upper row) and $20 \mu \mathrm{m}$ (lower row). (C) The morphology and distribution of microglia in the spinal cord did not change at any time point after hemorrhage $(P>0.05$, group effect by 2 -way ANOVA). Sections were taken from the level of lumbar 5 .

7 after hemorrhage (Figure 5A). TH increased the number of c-fos immunoreactive neurons in L4. In contrast, in the PLX-treated group, c-fos immunoreactivity remained at the Control level. We counted the number of c-fos-positive neurons in each group (Figure 5B; Control group, $n=4$; TH group, $n=4$; $\mathrm{TH}+\mathrm{PLX}$ group, $n=4$ ) and confirmed that the number of c-fos-positive neurons in L4 was significantly higher in the TH group than in the Control and TH + PLX groups. We further analyzed the proportion of c-fos-positive cells in L4 to those in L4 and L5a (Figure 5C). Again, the number of c-fos-positive cells was significantly higher in the TH group than in the TH + PLX and Control groups. These results indicate that neuronal activity in the S1 was altered by $\mathrm{TH}$ and that microglial depletion can prevent the generation of ectopic neuronal hyperactivity.

\section{Discussion}

In the present study, by using a mouse model of CPSP induced by a focal $\mathrm{TH}$, we showed that microglial depletion by PLX3397 effectively prevents allodynia development. Moreover, we discovered a critical time window of microglial involvement in the development of TH-induced allodynia. For effective attenuation of allodynia, microglia should be ablated in the initial phase after hemorrhage.

Despite increasing evidence indicating that microglia are key regulators of neuropathic pain induced by peripheral nerve injury (PNI), few studies have focused on microglial involvement in the pathophysiology of CPSP. In one study using a TH mouse model, minocycline treatment has been shown to ameliorate existing mechanical allodynia (19), suggesting microglial involvement in the maintenance of TH-induced allodynia, although the effect was only temporal ( 4 hours) (19). In contrast, our study revealed that the antiallodynic effect of PLX treatment in the initiation phase lasted for at least 2 weeks after stopping PLX administration. On the 
A

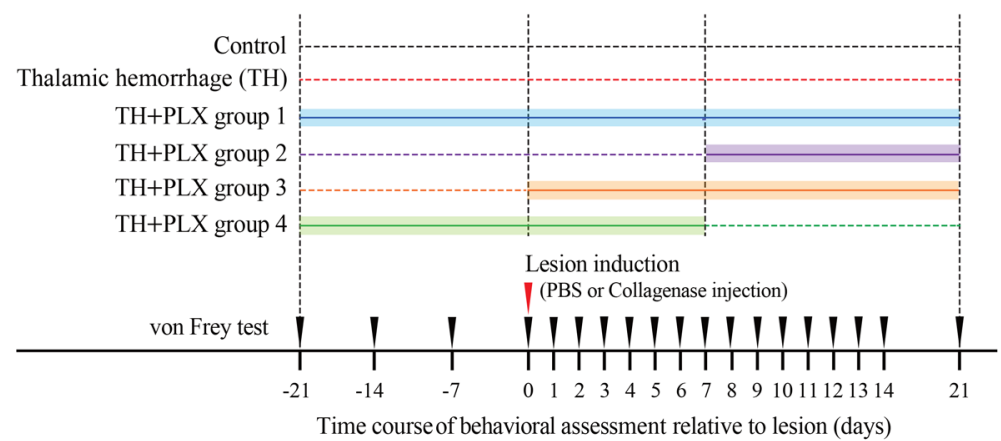

B
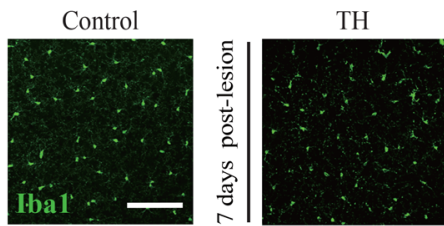

TH+PLX group 1

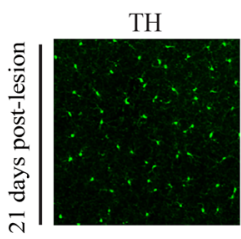

TH+PLX group 1
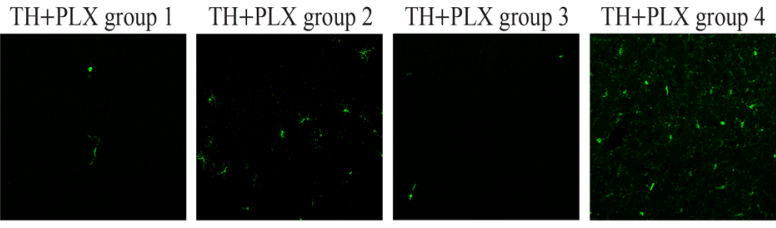

\section{E}

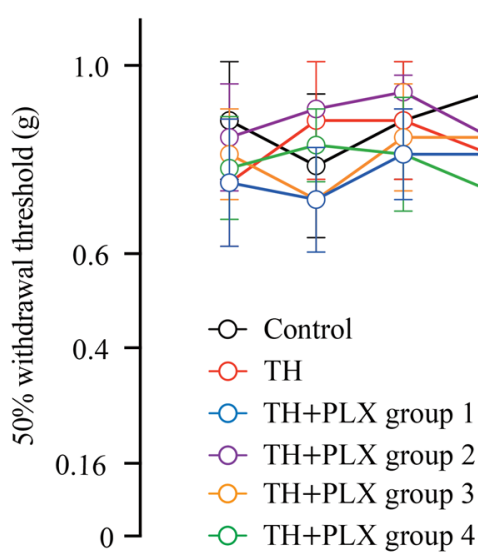

\begin{tabular}{|c|c|c|c|c|c|c|c|}
\hline$T$ & 1 & 1 & $T$ & $T$ & $T$ & 1 & 1 \\
\hline-21 & -14 & -7 & 0 & 4 & 7 & 14 & 21 \\
\hline
\end{tabular}

C

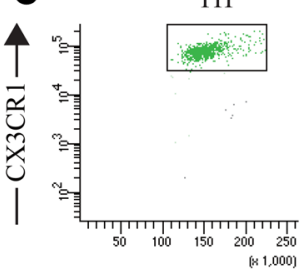

D

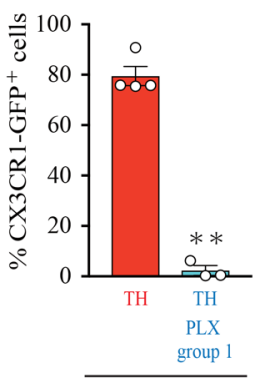

7 days post-lesion
$\mathrm{TH}+$ PLX-treatment

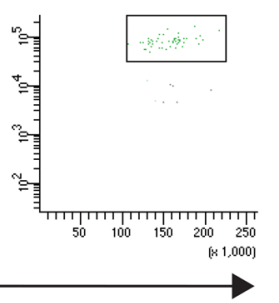

Figure 3. Microglial depletion prevented the development of mechanical allodynia induced by thalamic hemorrhage. (A) Schematic of the experimental design. Control group mice received PBS injection into the ventral posterolateral nucleus (VPL) $(n=4)$. Thalamic hemorrhage (TH) group mice received collagenase injection and were fed control chow. TH + PLX group 1 mice were treated with PLX3397 from 21 days before hemorrhage until 21 days after hemorrhage $(n=4)$. TH + PLX group 2: from days 7-21 $(n=4)$. TH + PLX group 3: from days 0-21 $(n=$ 4). $\mathrm{TH}+\mathrm{PLX}$ group 4: from 21 days before to 7 days after $\mathrm{TH}(n=5)$. (B) Iba-1 immunostaining of the S1 showed a robust decrease in microglial cells by PLX treatment. Scale bar: $100 \mu \mathrm{m}$. (C) Dot plots show the gating of CX3CR1 in each group. The plots were used to quantify the amount of microglia after TH. CX3CR $1^{+/ \text {ECFP }}$ mice were used in the flow cytometry experiment. (D) Flow cytometry quantification showed that the number of microglia in the group treated with PLX3397 was significantly reduced compared with that in the TH group on day $7\left(^{* *} P<0.01\right.$, unpaired $t$ test) and on day $21{ }^{* *} P<0.01,1$-way ANOVA followed by Dunnett's multiple comparisons test) after hemorrhage. (E) The 50\% withdrawal threshold in the TH group was significantly reduced after hemorrhage compared with the Control group $\left({ }^{*} P<0.01,{ }^{*} P<0.05\right.$, 2-way repeated measures ANOVA followed by Tukey's multiple comparisons test). $\mathrm{TH}+\mathrm{PLX}$ groups 1 and 4 , which started PLX treatment before lesion induction, exhibited a higher withdrawal threshold than the TH group did ${ }^{\# \# P} P<0.01$, ${ }^{\#} P<0.05$, 2-way repeated-measures ANOVA followed by Tukey's multiple comparisons test); however, TH + PLX group 2 and 3, which started PLX treatment after lesion induction, exhibited a lower withdrawal threshold, similar to the level exhibited by the TH group.

other hand, delayed PLX treatment had no effect on the existing allodynia. These results might be explained by the notion that microglial depletion in the initiation phase of allodynia has a potential disease-modifying effect. This assumption is supported by the observation that $\mathrm{TH}$-induced ectopic anatomical modification is suppressed by the PLX treatment (as discussed below). Considering the antiallodynic effect in the maintenance phase, the discrepancy between Hanada's study (19) and our study might come from the broad effect of minocycline. 
A

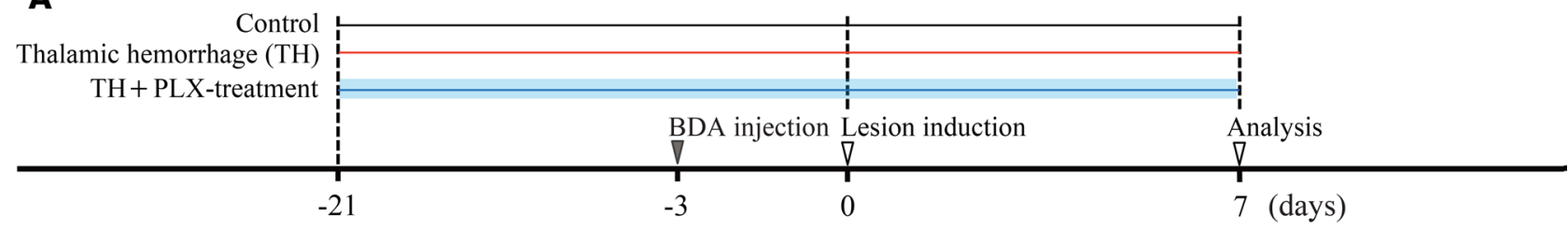

B

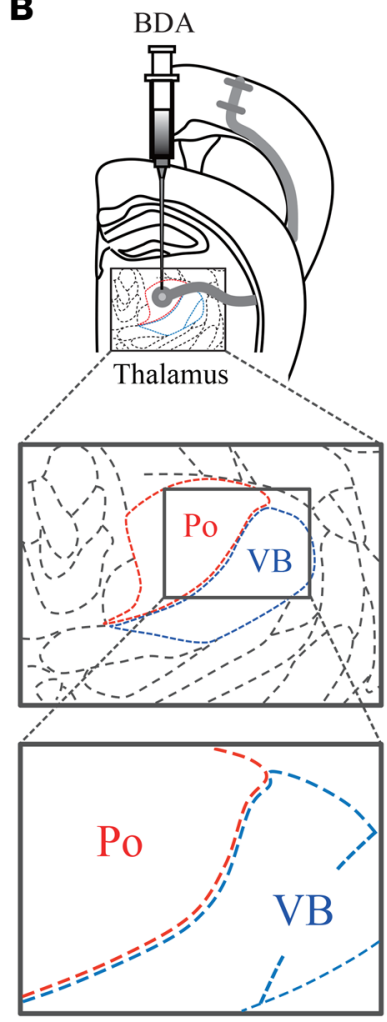

C

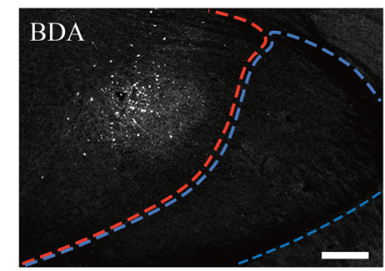

Bregma $-2.06 \mathrm{~mm}$
D
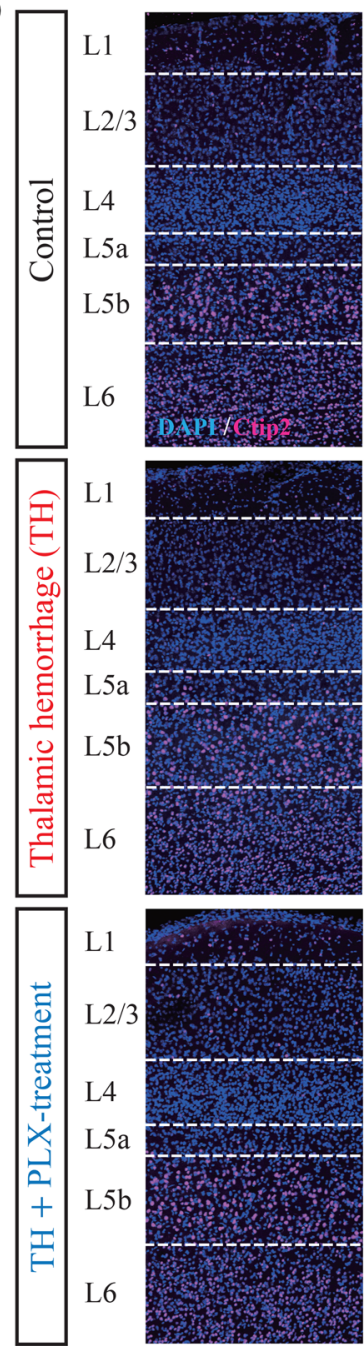
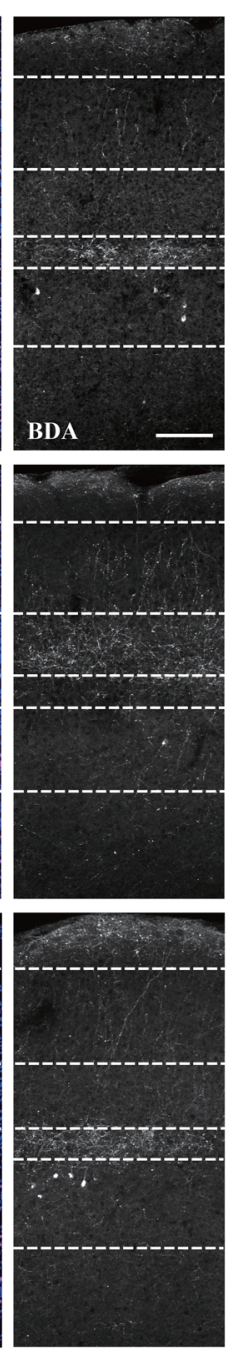

E

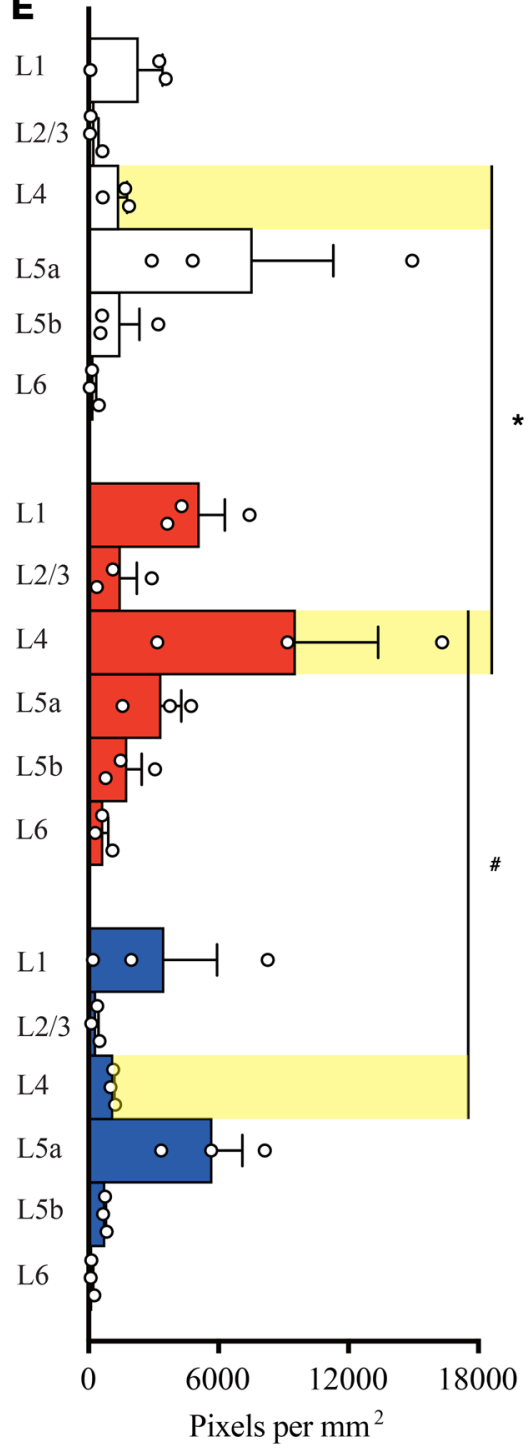

Figure 4. Thalamic hemorrhage induces abnormal formation of thalamocortical projections into layer $\mathbf{4}$ of the S1. (A) Experimental design and timeline. (B and C) Biotinylated dextran amines (BDA) injection into the posterior nucleus (Po) of the thalamus adjacent to the VB. Scale bar: $200 \mu \mathrm{m}$. (D and E) Distribution of BDA-labeled Po axons in each cortical layer of the S1. The fluorescence intensity (pixel per mm²) was higher in cortical layer 4 of the thalamic hemorrhage (TH) group than in that of the TH + PLX group and Control group $\left({ }^{*} P<0.01\right.$, layer 4 of TH group vs. layer 4 of Control group; ${ }^{*} P<0.01$, layer 4 of TH group vs. layer 4 of TH + PLX group. 2-way ANOVA followed by Tukey's multiple comparisons test. Scale bar: $200 \mu \mathrm{m}$.

Although minocycline is commonly used to halt microglial activity, it has a nonspecific effect on macrophages, T cells, neuronal cells (30-33), and even on astrocytes (34); all of them are suggested to be involved in the pathogenesis of neuropathic pain $(22,35-41)$. In contrast, treatment with a CSF1R inhibitor results in effective ablation of microglia with minor effect on other cells (25-28), which is also supported by our flow cytometry data, although we cannot fully exclude the possible influence of PLX3397 treatment on peripheral CSF1R-positive circulating myeloid cells. To elucidate the precise mechanism of microglial involvement in the development and maintenance of CPSP, future studies using more specific genetic tools will be needed $(22,42-44)$. 
A

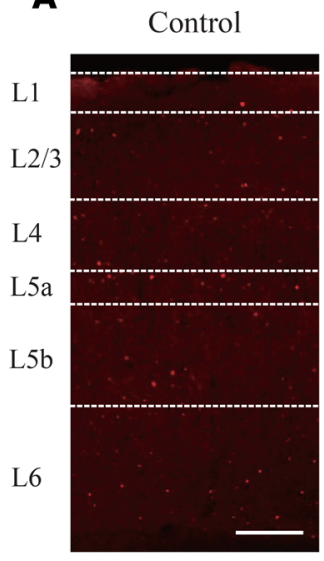

$\mathrm{TH}$

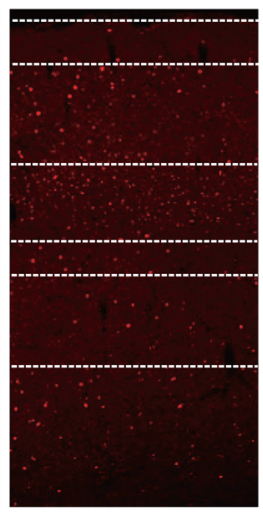

$\mathrm{TH}+\mathrm{PLX}$-treatment

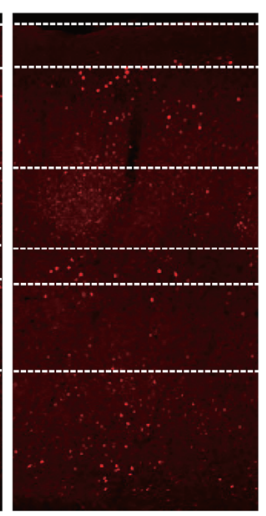

B

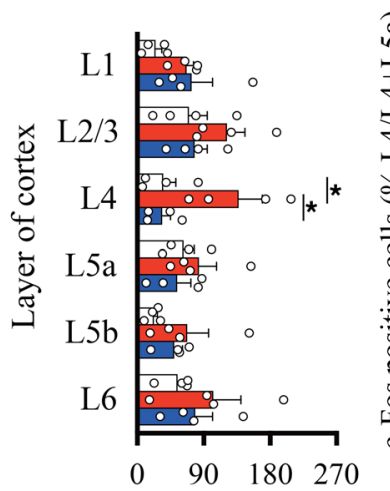

Ratio of c-Fos positive cells
C

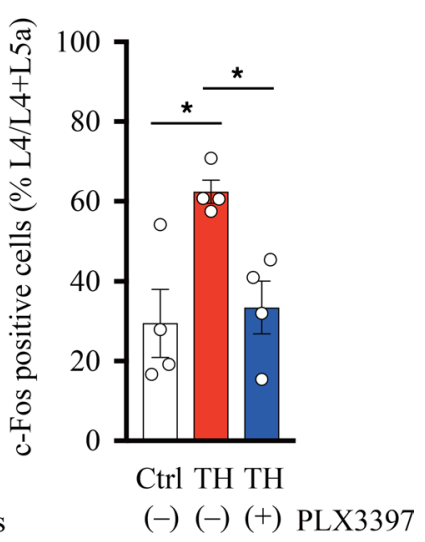

Figure 5. Microglial depletion prevents ectopic neuronal activity in the S1. (A) Representative images of coronal brain slices of S1 stained for c-fos on day 7 after hemorrhage. Borders of the cortical layers were identified based on the distribution of DAPI- and Ctip2-positive cells. Scale bar: $200 \mu \mathrm{m}$. (B) The number of c-fos-positive cells was higher in layer 4 in the thalamic hemorrhage (TH) group compared with that in the Control ${ }^{*} P<0.01,2$-way ANOVA followed by Tukey's multiple comparisons test) and TH + PLX groups ( ${ }^{*} P<0.01,2$-way ANOVA followed by Tukey's multiple comparisons test). (C) The ratio of c-fos-positive cells distributed in L4 to all the neurons counted within L4 and L5a. The percentage of c-fos-positive cells was higher in the TH group than in the TH + PLX group ( ${ }^{*} P<0.05$, 1-way ANOVA followed by Tukey's multiple comparisons test) and Control group $\left({ }^{*} P<0.01,1\right.$-way ANOVA followed by Tukey's multiple comparisons test).

Recently, sex dimorphism has been implicated in rodent models of neuropathic pain after PNI (45-47), and the difference is thought to be derived from variations in the immune response between males and females. In particular, although microglia play dominant roles in the development of allodynia after PNI in male mice, this is not the case in female mice; microglia inhibition by several glial inhibitors lead to the reversal of allodynia in male mice but not in female mice (45). On the other hand, in our CPSP model, we did not find any sex differences in the development of allodynia and in the effect of pharmacological depletion of microglia. Thus, we speculate that the pathophysiology of TH-induced allodynia may greatly differ from that of PNI-induced allodynia. Future studies will be needed to uncover the differences in the underlying mechanism of pain development among various conditions.By histological analysis, we observed microglial activation not only around the damaged VPL, but also in the ipsilesional S1, with little or no change in the contralesional cortex. This morphological change was observed coinciding with the onset of pain development. Furthermore, little or no change was observed in the microglia morphology and the regulation of microglia-related genes in the spinal cord, where the critical involvement of microglia in the pathogenesis of pain development is implicated after PNI (48). In this regard, Gritsch et al. showed that the spinal and peripheral components of the pain system do not contribute to the induction or maintenance of CPSP (49). Thus, we hypothesized that microglial activation in the S1 might have an undetermined role in the development of thalamic pain.

The somatosensory cortex may be involved in the pathophysiology of neuropathic pain $(1,5,50$, 51). However, the exact role of the $\mathrm{S} 1$ in pain development remains poorly understood. Moreover, at present, there is little evidence for the association of the S1 and CPSP development. In the present study, TH caused a marked increase in neuronal activity in the S1 as demonstrated by c-fos expression, suggesting that this structure is also involved in CPSP pathophysiology. Recent research has shown persistent hyperactivity of the S1 after PNI and its relevance to mechanical allodynia (52). Thus, different neuropathic pain conditions could share similar physiological mechanisms.

The noteworthy findings of our study are that the increased activity in the S1 is accompanied by the structural reorganization of the thalamocortical projection. In normal conditions, the S1 receives a dense axonal projection from the thalamic nucleus, including the VB and Po $(29,53)$. The VB receives inputs from the spinothalamic tract and mainly conveys tactile input $(29,54,55)$, while the Po mainly conveys nociceptive input $(29,54,56)$. We visualized the axonal projection from the Po to the $\mathrm{S} 1$ cortex in no-hemorrhage mice and confirmed that Po axons project to L1 and L5a, as previously reported $(29,57)$. Then, we tested the hemorrhagic animals; surprisingly, Po neurons changed their projection pattern. Po axons mainly branched within L4 in the S1, which normally receives inputs from the VB. 


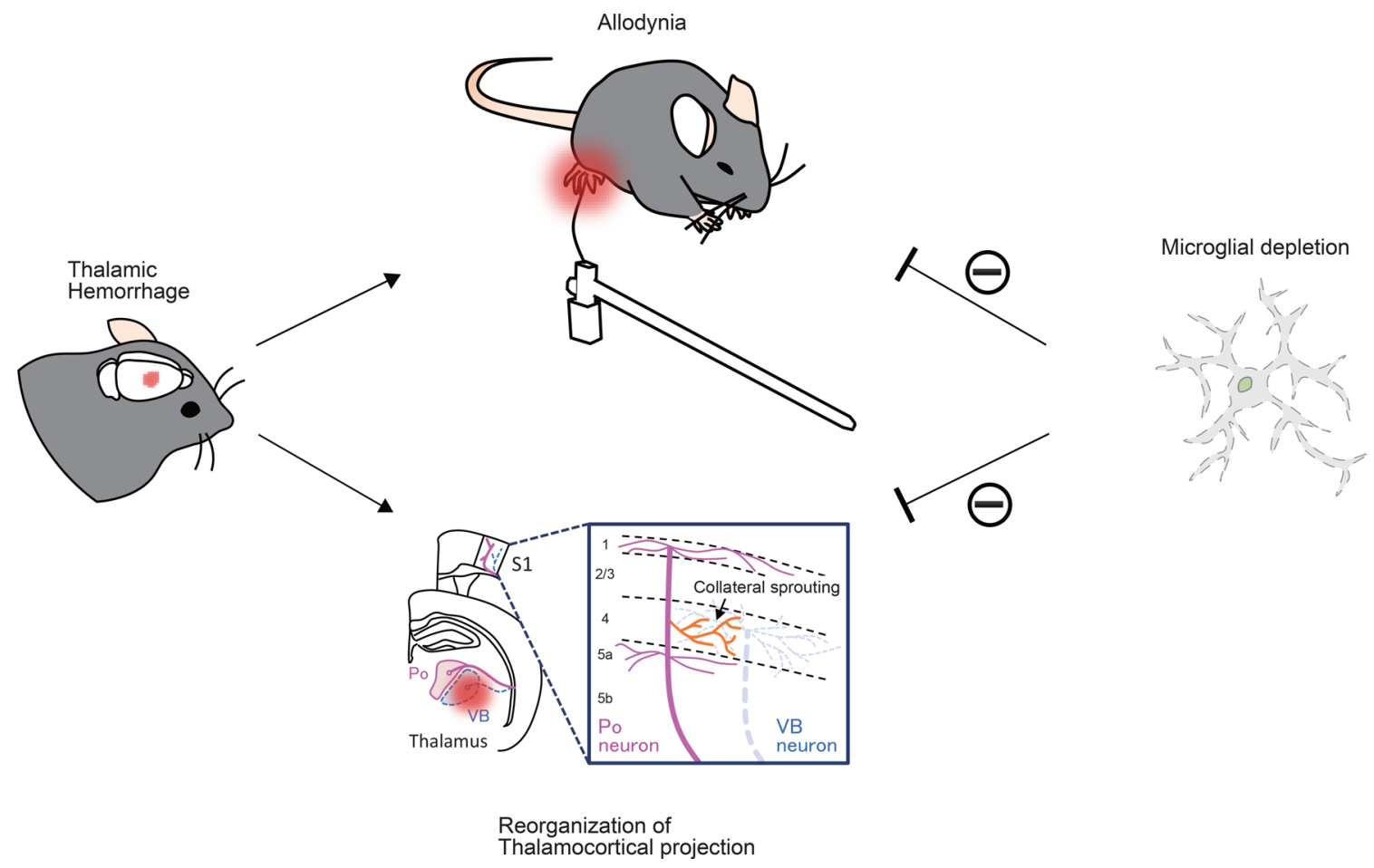

Figure 6. Schematic illustration of the findings. Thalamic hemorrhage-induced allodynia is accompanied by the aberrant reorganization of thalamocortical projection. Microglial ablation by the CSF1R inhibitor attenuates the development of allodynia and circuit reorganization.

This phenomenon might be explained as lesion-induced neural network reorganization to compensate for the destroyed thalamic projection. Regarding the developmental stage, genetic destruction of the VB at birth results in anatomical rewiring of Po projections onto the L4 neurons in the S1 to compensate for the lost function of the VB (29). Thus, the mechanism of compensatory axonal sprouting could be similar between the developmental stage and the repair phase after brain injury in adults.

We demonstrated that mechanical allodynia after $\mathrm{TH}$ is attenuated by microglial depletion; the development of allodynia is accompanied by neural hyperactivity in the S1 and the occurrence of ectopic axonal projection from the thalamus to the S1. Thus, we sought to determine whether microglial depletion can also reverse these functional and anatomical changes. Interestingly, enhanced neuronal activity in the S1 was completely abolished by PLX treatment. Moreover, ectopic Po projection to the L4 of S1 was attenuated by PLX treatment. These findings indicate that microglia promote the neural circuitry reorganization of the thalamocortical pathway that may contribute to CPSP development after hemorrhage in the thalamic nucleus. As discussed above, maladaptive plastic changes in the S1 may be important in the pathophysiology of neuropathic pain following PNI $(52,58)$. In particular, after PNI, structural and functional synaptic remodeling has been observed, and this plasticity may be involved in the development and maintenance of pain symptoms $(59,60)$. In addition, a cumulative body of evidence suggests that microglia are crucial regulators of neuronal/synaptic plasticity in health and disease $(18,61,62)$. Moreover, activated microglia in the spinal dorsal horn reportedly mediate aberrant excitability of neurons through a variety of plastic modifications of neuronal connections $(21,63)$. Thus, microglia may be likely to trigger maladaptive plasticity in the injured brain.

In the present study, we demonstrated that TH-induced allodynia was accompanied by the large-scale reorganization of the thalamocortical circuit and provided evidence that microglial ablation attenuates both the development of allodynia and circuit reorganization (Figure 6). Nevertheless, further studies are needed to prove the causal relationship between anatomical plasticity and behavioral symptoms (allodynia) and to reveal the underlying molecular and cellular mechanisms of microglial involvement in alterations in neuronal connectivity. We believe that our findings provide a framework for future research aimed at establishing a disease-modifying therapy for CPSP. 


\section{Methods}

\section{Subjects and group assignment}

A total of 142 male and 24 female C57BL/6J mice (aged 8-11 weeks, Japan SLC Inc.) and a total of 70 adult male CX3CR1 ${ }^{+/ E G F P}$ mice (aged 8-11 weeks from the Jackson Laboratory) were used in this study. The groups were as follows: (a) Control group (mice that received PBS as a sham operation), (b) TH group (mice that received collagenase injection), and (c) TH + PLX group (mice that received PLX3397 [Selleck Chemicals] treatment before or after TH). The timeline of PLX3397 administration in the TH + PLX groups is indicated in Figure 3A. No homozygous mice were used. Two to 3 mice were housed in standard cages with access to chow and water ad libitum under a 12-hour light-dark cycle with ambient temperature maintained at $24^{\circ} \mathrm{C}$.

\section{CPSP model}

Mice in the TH, PBS, and TH + PLX groups were anesthetized with a mixture of medetomidine hydrochloride (0.3 mg/kg; Domitor, Orion Pharma), midazolam (4 mg/kg; Dormicum, Astellas), and butorphanol (5 mg/kg; Vetorphale, Meiji Seika Pharma) and placed on a panel heater in a stereotaxic frame during surgery. We employed an intracerebral collagenase injection model. Collagenase enzymatically lyses the extracellular matrix, causing disruption of brain capillaries and consequent active bleeding (64-66). Of note, matrix metallopeptidase (MMP) can directly cause microglial activation and is involved in the pathogenesis of neuropathic pain after PNI (41). TH was induced by injecting $0.025 \mathrm{U}$ collagenase type IV (MilliporeSigma, C5138) dissolved in PBS at the stereotaxic coordinate of the VPL in the TH and TH + PLX groups. Based on a previous study (19), a custom-ordered cannula was inserted at slightly modified coordinates as follows: posterior $1.1 \mathrm{~mm}$ to bregma, lateral to the midline from $1.5 \mathrm{~mm}$, and ventral to the surface of the cortex from $3.5 \mathrm{~mm}$. A total of $25 \mathrm{~nL}$ of collagenase was released into the thalamic VB through a tube, and the cannula was connected to $25 \mu \mathrm{L}$ gastight syringes (Hamilton, 80200) at a constant rate of $5 \mathrm{~nL} /$ minute using an infusion pump (Eicom, ESP-32). An equivalent volume of PBS was injected into the right VB for the Control group.

\section{Behavioral tests}

von Frey test. All mice were habituated to a plastic box with a perforated metal floor for 15-30 minutes before the assessments. Data were quantified from 6 male mice from the $\mathrm{TH}$ and Control groups to assess the hemorrhage effect, and another group of male mice $(n=4-5)$ from the TH, Control, and $4 \mathrm{TH}+$ PLX groups was used to assess the effect of PLX treatment. A range force of mechanical stimulation using 0.02-2 g monofilaments (Semmes-Weinstein von Frey Anesthesiometer; Muromachi Kikai) was applied to the bilateral hind paws of each mouse, and a $50 \%$ withdrawal threshold was determined by the up-down method (67). The von Frey test was conducted daily from the time of hemorrhage induction for 7 consecutive days; thereafter, it was measured once a week until 140 days after injection. The von Frey test was also performed in female mice to test whether there was a sex difference (Control group $=6, \mathrm{TH}$ group $=8, \mathrm{TH}+\mathrm{PLX}$ group $=10$ ).

Neurological examination. Neurological deficits after hemorrhage were assessed according to a 4-point-scale method (68). Scores were obtained from 6 TH mice and 5 Control mice. Specifically, grade 0 was assigned when there were no observable deficits; grade 1 was assigned for forelimb flexion; grade 2 was assigned when a decreased resistance to lateral push without circling was detected; and grade 3 was noted when a mouse exhibited behavior very similar to that for grade 2 but showed no circling. Grade 0 was normal, grade 1 was moderate, and grades 2 and 3 were considered severe neurological deficits induced by the lesion.

Ladder walk test. Motor function was assessed by the ladder walk test (69). The number of slips was counted on a ladder walk ( $1 \mathrm{~m}$ in length) in which the step bars randomly changed in each trial (70). The number of slips was divided by the total number of steps. Each mouse was given 3 trials on each assessment day, and results were quantified from 5-6 mice in the TH and Control groups.

Rotarod test. Motor coordination in mice was assessed by the rotarod test. Five to 6 mice in both the $\mathrm{TH}$ and Control groups were acclimated to a rotating rod (a diameter of $30 \mathrm{~mm}$ ) for at least 10-20 minutes before data acquisition. The rod was set to accelerate from 4-40 rpm. The duration was recorded until a mouse fell from the rod or until a mouse rotated with the rod without directional running on the rotating rod. The test was repeated 3 times a day. 


\section{Anterograde labeling with BDA}

To label thalamocortical axons from the Po, we injected anterograde tracer BDA into the thalamus in 3 mice in the TH, Control, and TH + PLX groups. C57BL/6 mice were anesthetized with a mixture of medetomidine $(0.3$ $\mathrm{mg} / \mathrm{kg}$ ), midazolam ( $4 \mathrm{mg} / \mathrm{kg})$, and butorphanol $(5 \mathrm{mg} / \mathrm{kg}$ ) and placed in a stereotaxic frame. A custom-ordered cannula attached to gastight syringes (Hamilton, 80200) containing 10\% BDA (Invitrogen, D1820) was connected to the infusion pump. The cannula was slowly inserted into the Po of the thalamus (at the coordinates of anteriorposterior to bregma $-2.0 \mathrm{~mm}$, lateral to the midline from $1.25 \mathrm{~mm}$, ventral to the surface of the cortex $3.0 \mathrm{~mm}$; Figure $4 \mathrm{~B}$ ), and the BDA was delivered over 4 minutes at a flow rate of $50 \mu \mathrm{L} /$ minute. The TH, TH + PLX, and PBS groups received either collagenase or PBS in the thalamus 3 days after the BDA injection. The TH + PLX group started to receive PLX3397 for 21 days before the collagenase injection (Figure 4A). Animals were sacrificed 7 days after collagenase/PBS injection and 10 days after BDA injection.

\section{Microglial ablation with oral administration of CSF1R inhibitor}

Microglia in the CNS were eliminated with oral administration of selective CSF1R inhibitor PLX3397 (25). A total of 67 mice received free access to PLX3397 chow as the sole food source in the home cage. The administration period for PLX3397 is indicated in Figure 3A. PLX3397 was purchased from Selleck Chemicals, and it was formulated with Research Diets into AIN-76A chow at $0.029 \%$. Mice treated with PLX3397 were also housed in standard cages with free access to PLX-containing chow only and water.

\section{Flow cytometry}

The number of microglia in the brain after hemorrhage was analyzed using flow cytometry. A total of 3-5 mice in the TH, PBS, and the $4 \mathrm{TH}+$ PLX groups were perfused with cold PBS. The timeline of the 4 groups that underwent PLX treatment is shown in Figure 3A. The right hemisphere was then digested with $0.1 \%$ collagenase $\mathrm{D}$ (Roche, 11088866001 ) containing $2.5 \mathrm{mM}$ calcium chloride at $37^{\circ} \mathrm{C}$ for 30 minutes. The cell suspension was passed through a $70-\mu \mathrm{m}$ cell strainer and was isolated by centrifugation at $780 \mathrm{~g}$ for 30 minutes with 30\% and 70\% Percoll (GE Healthcare, 17-0891-01). The cells isolated from the interfaces of $30 \% / 70 \%$ Percoll gradients were stained with a fluorescently conjugated antibody (BioLegend; PE-CD11b, 301306; Pacific Blue-CD45, 103126; APC-Ly6C, 128016; Brilliant Violet-Ly6G, 127639) for 30 minutes at $4^{\circ} \mathrm{C}$ in a light-shielded state. The cells were gated according to size and granularity based on forward scatter and side scatter properties using FACS Aria II (BD Biosciences). The GFP-positive cells quantified for the CX3CR1 $1^{+/ E G F P}$ mice using FACS Diva Software.

\section{Histological analysis}

A total of 84 mice were euthanized by overdose with a mixture of medetomidine hydrochloride $(0.3$ $\mathrm{mg} / \mathrm{kg}$ ), midazolam (4 mg/kg), and butorphanol $(5 \mathrm{mg} / \mathrm{kg}$ ) and were perfused with PBS and $4 \%$ paraformaldehyde (Nacalai Tesque, 02890-45). After perfusion, the brain and spinal cord were removed separately and further fixed with $4 \%$ paraformaldehyde for 2 hours at $4{ }^{\circ} \mathrm{C}$. Next, the brain and spinal cord were transferred to $15 \%$ sucrose/PBS for 2 hours, subsequently transfered to $30 \%$ sucrose/PBS, stored overnight at $4^{\circ} \mathrm{C}$, and sectioned at $50-\mu \mathrm{m}$ and $30-\mu \mathrm{m}$ thickness, respectively, on a cryostat. For IHC, a series of brain sections (coronal, from $0.62 \mathrm{~mm}$ anterior to $2.54 \mathrm{~mm}$ posterior relative to bregma) were permeabilized in PBS containing 0.3\% Triton X-100 and then blocked with 3\% normal goat serum and $1 \%$ BSA for 1 hour at room temperature. The sections were incubated with anti-Iba-1 (1/1000, Wako, 019-19741), anti-c-fos (1/1000, MilliporeSigma, ABE457), and anti-Ctip2 (1/500, Abcam, ab18456). Similarly, a series of spinal cord sections from lumbar cord L4-L6 were stained with anti-Iba-1. After 3 washes with PBS, the sections were incubated with DAPI and each secondary antibody (1/500, Invitrogen, goat anti-rabbit, A11008 and A11011; goat anti-rat, A21247) in PBS for 90 minutes at room temperature. For visualizing the BDA-labeled thalamus axon, the sections were incubated with Alexa Fluor 568 streptavidin (1/400, Invitrogen, S11226) in PBS for 90 minutes. The sections were washed 3 times with PBS and mounted on slides. Images were taken with a confocal microscope (Olympus, FV1200). To analyze the lesion volume after collagenase injection, we stained all sections with Cresyl violet and collected images of the sections. The lesion volumes of the thalamus and BDA-labeled thalamic axons were quantified using Image $(\mathrm{NIH})$, and c-fos expression in each layer of the S1 was analyzed by Adobe Photoshop CC 2017. 


\section{Quantitative PCR}

Total RNA was isolated from bilateral S1, thalamus, and lumbar cord (L4-L6) tissue of TH group and from right S1, right thalamus, and left lumbar cord (L4-L6) tissue of Control group, with TRIzol reagent (Invitrogen) and RNeasy mini kit (QIAGEN) on day 4 after PBS or collagenase injection into the thalamus $(n=3-5)$. cDNA was synthesized from $15 \mu \mathrm{g}$ of total RNA using the High Capacity cDNA Reverse Transcription Kit (Applied Biosystems). To detect amplification in the Quant Studio 7 Flex Real-Time PCR system (Applied Biosystems), real-time PCR was performed using Fast SYBR Green Mater Mix (Applied Biosystems) at $95^{\circ} \mathrm{C}$ for 20 seconds, followed by 40 cycles of $95^{\circ} \mathrm{C}$ for 3 seconds and $60^{\circ} \mathrm{C}$ for 30 seconds. The gene expression levels were normalized by the geometric mean of Gapdh and Rps 18 expression levels after being calculated by the relative standard curve method. The average value of the Control group was set as 1 . The primer pair used were Gapdh, 5' - TGTGTCCGTCGTGGATCTGA - 3' and 5' - TTGCTGTTGAAGTCGCAGGAG - 3'; Rps 18, 5' - CATGCAGAACCCACGACAGTA - 3' and 5' - CCTCACGCAGCTTGTTGTCTA - 3'; Tnfa , 5' CTGTAGCCCACGTCGTAGC - 3' and 5' - TTGAGATCCATGCCGTTG - 3'; Il-1 $1 \beta$, 5' - CGCAGCAGCACATCAACAAG - $3^{\prime}$ and $5^{\prime}$ - GTGCTCATGTCCTCATCCTG - 3'; Il-6, 5' - CTGCAAGAGACTTCCATCCAGTT - $3^{\prime}$ and $5^{\prime}$ - AAGTAGGGAAGGCCGTGGTT - 3'; Il-10, 5' - GCTCTTACTGACTGGCATGAG - $3^{\prime}$ and $5^{\prime}$ - CGCAGCTCTAGGAGCATGTG - $3^{\prime} ;$ Bdnf, $5^{\prime}$ - CAGGTTCGAGAGGTCTGACG - $3^{\prime}$ and 5' - AAGTGTACAAGTCCGCGTCC - 3'; Trem2, 5' - GGAACCGTCACCATCACTCT - 3' and 5' - ATGCTGGCTGCAAGAAACTT - 3'; and Cd68, 5' - ACTGGTGTAGCCTAGCTGGT - 3' and 5' - CCTTGGGCTATAAGCGGTCC - $3^{\prime}$.

Statistics. Statistical analysis was performed using GraphPad Prism 7 software, and the analysis results are shown in the figure legends. Data from 3 or more categorical, independent groups were compared using 1-way or 2-way ANOVA, followed by a post hoc test (Sidac's multiple comparison test, Dunnett's multiple comparison test, and Tukey's multiple comparison test. Comparisons between the 2 groups were made using unpaired 2-tailed $t$ tests. A $P$ value of less than 0.05 was considered significant in all statistical tests. Data are shown as the mean \pm SEM.

Study approval. All procedures were approved by the Guidelines for the Care and Use of Laboratory Animals of Osaka University Graduate School of Medicine.

\section{Author contributions}

$\mathrm{SH}, \mathrm{TI}, \mathrm{MH}, \mathrm{MN}$, and TY designed and contributed to the original conception. SH conducted all the experiments. HT performed IHC for the spinal cord. SH, TI, MH, MN, and TY wrote the manuscript.

\section{Acknowledgments}

We are grateful to Tomomi Inai in the Center for Medical Research and Education, Graduate School of Medicine, Osaka University, for technical assistance with flow cytometry analysis. We thank Nao Shibuya for discussions and comments on the paper. This work was supported by the Japan Society for the promotion of Science (JSPS) KAKENHI grants A17H061780 to TY, A16J40050 to MH, and A18J118340 to SH, as well as AMED-CREST (18gm1210005h0001) to TY.

Address correspondence to: Toshihide Yamashita and Takahide Itokazu, 2-2 Yamadaoka, Suita, Osaka 565-0871, Japan. Phone: 81.6.6879.3661; Email: yamashita@molneu.med.osaka-u.ac.jp (TY), t.itokazu@ molneu.med.osaka-u.ac.jp (TI).

MH's current address is: Biogen, 4-1, Nihonbashi 1-chome, Chuo-ku, Tokyo, Japan.

1. Klit H, Finnerup NB, Jensen TS. Central post-stroke pain: clinical characteristics, pathophysiology, and management. Lancet Neurol. 2009;8(9):857-868.

2. Andersen G, Vestergaard K, Ingeman-Nielsen M, Jensen TS. Incidence of central post-stroke pain. Pain. 1995;61(2):187-193.

3. Balami JS, Chen RL, Grunwald IQ, Buchan AM. Neurological complications of acute ischaemic stroke. Lancet Neurol. 2011;10(4):357-371

4. Hosomi K, Seymour B, Saitoh Y. Modulating the pain network--neurostimulation for central poststroke pain. Nat Rev Neurol. 2015;11(5):290-299.

5. Morishita T, Inoue T. Brain Stimulation Therapy for Central Post-Stroke Pain from a Perspective of Interhemispheric Neural Network Remodeling. Front Hum Neurosci. 2016;10:166

6. Chen G, Zhang YQ, Qadri YJ, Serhan CN, Ji RR. Microglia in Pain: Detrimental and Protective Roles in Pathogenesis and Resolution of Pain. Neuron. 2018;100(6):1292-1311. 
7. Ransohoff RM, Cardona AE. The myeloid cells of the central nervous system parenchyma. Nature. 2010;468(7321):253-262. 8. Butovsky O, Weiner HL. Microglial signatures and their role in health and disease. Nat Rev Neurosci. 2018;19(10):622-635.

9. Davalos D, et al. ATP mediates rapid microglial response to local brain injury in vivo. Nat Neurosci. 2005;8(6):752-758.

10. Hoshiko M, Arnoux I, Avignone E, Yamamoto N, Audinat E. Deficiency of the microglial receptor CX3CR1 impairs postnatal functional development of thalamocortical synapses in the barrel cortex. J Neurosci. 2012;32(43):15106-15111.

11. Li Q, Barres BA. Microglia and macrophages in brain homeostasis and disease. Nat Rev Immunol. 2018;18(4):225-242.

12. Nimmerjahn A, Kirchhoff F, Helmchen F. Resting microglial cells are highly dynamic surveillants of brain parenchyma in vivo. Science. 2005;308(5726):1314-1318.

13. Paolicelli RC, et al. Synaptic pruning by microglia is necessary for normal brain development. Science. 2011;333(6048):1456-1458.

14. Salter MW, Beggs S. Sublime microglia: expanding roles for the guardians of the CNS. Cell. 2014;158(1):15-24.

15. Heneka MT, McManus RM, Latz E. Inflammasome signalling in brain function and neurodegenerative disease. Nat Rev Neurosci. 2018;19(10):610-621.

16. Milligan ED, Watkins LR. Pathological and protective roles of glia in chronic pain. Nat Rev Neurosci. 2009;10(1):23-36.

17. Iadecola C, Anrather J. The immunology of stroke: from mechanisms to translation. Nat Med. 2011;17(7):796-808.

18. Salter MW, Stevens B. Microglia emerge as central players in brain disease. Nat Med. 2017;23(9):1018-1027.

19. Hanada T, Kurihara T, Tokudome M, Tokimura H, Arita K, Miyata A. Development and pharmacological verification of a new mouse model of central post-stroke pain. Neurosci Res. 2014;78:72-80.

20. Deuis JR, Dvorakova LS, Vetter I. Methods Used to Evaluate Pain Behaviors in Rodents. Front Mol Neurosci. 2017;10:284

21. Inoue K, Tsuda M. Microglia in neuropathic pain: cellular and molecular mechanisms and therapeutic potential. Nat Rev Neurosci. 2018;19(3):138-152.

22. Peng J, et al. Microglia and monocytes synergistically promote the transition from acute to chronic pain after nerve injury. Nat Commun. 2016;7:12029.

23. Tsuda M, et al. P2X4 receptors induced in spinal microglia gate tactile allodynia after nerve injury. Nature. 2003;424(6950):778-783.

24. Wasserman JK, Koeberle PD. Development and characterization of a hemorrhagic rat model of central post-stroke pain. Neuroscience. 2009;161(1):173-183.

25. Elmore MR, et al. Colony-stimulating factor 1 receptor signaling is necessary for microglia viability, unmasking a microglia progenitor cell in the adult brain. Neuron. 2014;82(2):380-397.

26. Valdearcos M, Robblee MM, Benjamin DI, Nomura DK, Xu AW, Koliwad SK. Microglia dictate the impact of saturated fat consumption on hypothalamic inflammation and neuronal function. Cell Rep. 2014;9(6):2124-2138.

27. Mok S, et al. Inhibition of CSF-1 receptor improves the antitumor efficacy of adoptive cell transfer immunotherapy. Cancer Res. 2014;74(1):153-161

28. Tanabe S, Saitoh S, Miyajima H, Itokazu T, Yamashita T. Microglia suppress the secondary progression of autoimmune encephalomyelitis. Glia. 2019;67(9):1694-1704

29. Pouchelon G, et al. Modality-specific thalamocortical inputs instruct the identity of postsynaptic L4 neurons. Nature. 2014;511(7510):471-474.

30. Hellwig S, Heinrich A, Biber K. The brain's best friend: microglial neurotoxicity revisited. Front Cell Neurosci. $2013 ; 7: 71$.

31. Jiao X, Peng Y, Yang L. Minocycline protects retinal ganglion cells after optic nerve crush injury in mice by delaying autophagy and upregulating nuclear factor-кB2. Chin Med J. 2014;127(9):1749-1754.

32. Bastos LF, de Oliveira AC, Watkins LR, Moraes MF, Coelho MM. Tetracyclines and pain. Naunyn Schmiedebergs Arch Pharmacol. 2012;385(3):225-241.

33. Yong VW, Wells J, Giuliani F, Casha S, Power C, Metz LM. The promise of minocycline in neurology. Lancet Neurol. 2004;3(12):744-751.

34. Garwood CJ, Pooler AM, Atherton J, Hanger DP, Noble W. Astrocytes are important mediators of A $\beta$-induced neurotoxicity and tau phosphorylation in primary culture. Cell Death Dis. 2011;2:e167.

35. Vallejo R, Tilley DM, Vogel L, Benyamin R. The role of glia and the immune system in the development and maintenance of neuropathic pain. Pain Pract. 2010;10(3):167-184.

36. Moehring F, Halder P, Seal RP, Stucky CL. Uncovering the Cells and Circuits of Touch in Normal and Pathological Settings. Neuron. 2018;100(2):349-360.

37. Luo X, et al. Macrophage Toll-like Receptor 9 Contributes to Chemotherapy-Induced Neuropathic Pain in Male Mice. J Neurosci. 2019;39(35):6848-6864

38. Cobos EJ, et al. Mechanistic Differences in Neuropathic Pain Modalities Revealed by Correlating Behavior with Global Expression Profiling. Cell Rep. 2018;22(5):1301-1312.

39. Li T, Chen X, Zhang C, Zhang Y, Yao W. An update on reactive astrocytes in chronic pain. J Neuroinflammation. 2019;16(1):140.

40. Gao YJ, et al. JNK-induced MCP-1 production in spinal cord astrocytes contributes to central sensitization and neuropathic pain. J Neurosci. 2009;29(13):4096-4108

41. Kawasaki Y, et al. Distinct roles of matrix metalloproteases in the early- and late-phase development of neuropathic pain. Nat Med. 2008;14(3):331-336

42. Kaiser T, Feng G. Tmem119-EGFP and Tmem119-CreERT2 Transgenic Mice for Labeling and Manipulating Microglia. eNeuro. 2019;6(4):ENEURO.0448-18.2019.

43. Parkhurst CN, et al. Microglia promote learning-dependent synapse formation through brain-derived neurotrophic factor. Cell. 2013;155(7):1596-1609.

44. Ginhoux F, et al. Fate mapping analysis reveals that adult microglia derive from primitive macrophages. Science. 2010;330(6005):841-845.

45. Sorge RE, et al. Different immune cells mediate mechanical pain hypersensitivity in male and female mice. Nat Neurosci. 2015;18(8):1081-1083.

46. Sorge RE, Totsch SK. Sex Differences in Pain. J Neurosci Res. 2017;95(6):1271-1281.

47. Del Rivero T, Fischer R, Yang F, Swanson KA, Bethea JR. Tumor necrosis factor receptor 1 inhibition is therapeutic for neuropathic pain in males but not in females. Pain. 2019;160(4):922-931. 
48. Guan Z, et al. Injured sensory neuron-derived CSF1 induces microglial proliferation and DAP12-dependent pain. Nat Neurosci. 2016;19(1):94-101.

49. Gritsch S, Bali KK, Kuner R, Vardeh D. Functional characterization of a mouse model for central post-stroke pain. Mol Pain. 2016;12:1744806916629049.

50. Flor H, Nikolajsen L, Staehelin Jensen T. Phantom limb pain: a case of maladaptive CNS plasticity? Nat Rev Neurosci. 2006;7(11):873-881.

51. Kuner R, Flor H. Structural plasticity and reorganisation in chronic pain. Nat Rev Neurosci. 2017;18(2):113.

52. Cichon J, Blanck TJJ, Gan WB, Yang G. Activation of cortical somatostatin interneurons prevents the development of neuropathic pain. Nat Neurosci. 2017;20(8):1122-1132.

53. Wimmer VC, Bruno RM, de Kock CP, Kuner T, Sakmann B. Dimensions of a projection column and architecture of VPM and POm axons in rat vibrissal cortex. Cereb Cortex. 2010;20(10):2265-2276.

54. Frangeul L, et al. Specific activation of the paralemniscal pathway during nociception. Eur J Neurosci. 2014;39(9):1455-1464

55. Yu C, Derdikman D, Haidarliu S, Ahissar E. Parallel thalamic pathways for whisking and touch signals in the rat. PLoS Biol. 2006;4(5):e124.

56. Masri R, Quiton RL, Lucas JM, Murray PD, Thompson SM, Keller A. Zona incerta: a role in central pain. J Neurophysiol. 2009;102(1):181-191.

57. Ohno S, et al. A morphological analysis of thalamocortical axon fibers of rat posterior thalamic nuclei: a single neuron tracing study with viral vectors. Cereb Cortex. 2012;22(12):2840-2857.

58. Kim SK, Eto K, Nabekura J. Synaptic structure and function in the mouse somatosensory cortex during chronic pain: in vivo two-photon imaging. Neural Plast. 2012;2012:640259.

59. Kim SK, Nabekura J. Rapid synaptic remodeling in the adult somatosensory cortex following peripheral nerve injury and its association with neuropathic pain. J Neurosci. 2011;31(14):5477-5482

60. Kim W, Kim SK, Nabekura J. Functional and structural plasticity in the primary somatosensory cortex associated with chronic pain. J Neurochem. 2017;141(4):499-506.

61. Rogers JT, et al. CX3CR1 deficiency leads to impairment of hippocampal cognitive function and synaptic plasticity. $J$ Neurosci. 2011;31(45):16241-16250.

62. Sipe GO, Lowery RL, Tremblay MÈ, Kelly EA, Lamantia CE, Majewska AK. Microglial P2Y12 is necessary for synaptic plasticity in mouse visual cortex. Nat Commun. 2016;7:10905

63. Liu Y, et al. TNF- $\alpha$ Differentially Regulates Synaptic Plasticity in the Hippocampus and Spinal Cord by Microglia-Dependent Mechanisms after Peripheral Nerve Injury. J Neurosci. 2017;37(4):871-881.

64. Krafft PR, et al. Modeling intracerebral hemorrhage in mice: injection of autologous blood or bacterial collagenase. $J$ Vis Exp. 2012;(67):e4289.

65. Rosenberg GA, Mun-Bryce S, Wesley M, Kornfeld M. Collagenase-induced intracerebral hemorrhage in rats. Stroke. 1990;21(5):801-807.

66. MacLellan CL, et al. Intracerebral hemorrhage models in rat: comparing collagenase to blood infusion. J Cereb Blood Flow Metab. 2008;28(3):516-525.

67. Chaplan SR, Bach FW, Pogrel JW, Chung JM, Yaksh TL. Quantitative assessment of tactile allodynia in the rat paw. JNeurosci Methods. 1994;53(1):55-63.

68. Bederson JB, Pitts LH, Tsuji M, Nishimura MC, Davis RL, Bartkowski H. Rat middle cerebral artery occlusion: evaluation of the model and development of a neurologic examination. Stroke. 1986;17(3):472-476.

69. Metz GA, Whishaw IQ. The ladder rung walking task: a scoring system and its practical application. J Vis Exp. 2009;(28):e1204

70. Ueno M, Hayano Y, Nakagawa H, Yamashita T. Intraspinal rewiring of the corticospinal tract requires target-derived brain-derived neurotrophic factor and compensates lost function after brain injury. Brain. 2012;135(Pt 4):1253-1267. 